\title{
Modulation of Th1/Th2 Immune Responses by Killed Propionibacterium acnes and Its Soluble Polysaccharide Fraction in a Type I Hypersensitivity Murine Model: Induction of Different Activation Status of Antigen-Presenting Cells
}

\author{
Carla Cristina Squaiella-Baptistão, ${ }^{1}$ Daniela Teixeira, ${ }^{2}$ Juliana Sekeres Mussalem, ${ }^{2}$ \\ Mayari Eika Ishimura, ${ }^{2}$ and Ieda Maria Longo-Maugéri ${ }^{2}$ \\ ${ }^{1}$ Laboratório de Imunoquímica, Instituto Butantan, Avenida Vital Brazil 1500, Prédio Novo, $2{ }^{\circ}$ Andar, Butantã, \\ 05503-900 São Paulo, SP, Brazil \\ ${ }^{2}$ Disciplina de Imunologia, Departamento de Microbiologia, Imunologia e Parasitologia, Universidade Federal de São Paulo, \\ Rua Botucatu 862, Edifício de Ciências Biomédicas, $4^{\circ}$ Andar, Vila Clementino, 04023-062 São Paulo, SP, Brazil \\ Correspondence should be addressed to Ieda Maria Longo-Maugéri; imaugeri@unifesp.br
}

Received 5 September 2014; Revised 25 October 2014; Accepted 25 October 2014

Academic Editor: Oscar Bottasso

Copyright (c) 2015 Carla Cristina Squaiella-Baptistão et al. This is an open access article distributed under the Creative Commons Attribution License, which permits unrestricted use, distribution, and reproduction in any medium, provided the original work is properly cited.

\begin{abstract}
Propionibacterium acnes (P. acnes) is a gram-positive anaerobic bacillus present in normal human skin microbiota, which exerts important immunomodulatory effects, when used as heat- or phenol-killed suspensions. We previously demonstrated that heatkilled P. acnes or its soluble polysaccharide (PS), extracted from the bacterium cell wall, suppressed or potentiated the Th2 response to ovalbumin (OVA) in an immediate hypersensitivity model, depending on the treatment protocol. Herein, we investigated the mechanisms responsible for these effects, using the same model and focusing on the activation status of antigen-presenting cells (APCs). We verified that higher numbers of APCs expressing costimulatory molecules and higher expression levels of these molecules are probably related to potentiation of the Th2 response to OVA induced by $P$. acnes or PS, while higher expression of toll-like receptors (TLRs) seems to be related to Th2 suppression. In vitro cytokines production in cocultures of dendritic cells and T lymphocytes indicated that $P$. acnes and PS seem to perform their effects by acting directly on APCs. Our data suggest that $P$. acnes and PS directly act on APCs, modulating the expression of costimulatory molecules and TLRs, and these differently activated APCs drive distinct T helper patterns to OVA in our model.
\end{abstract}

\section{Introduction}

Propionibacterium acnes ( $P$. acnes) is a gram-positive anaerobic bacillus present in normal human skin microbiota [1] but also implicated in inflammatory diseases, such as acne vulgaris [2] and sarcoidosis [3]. An important property of this bacterium is its immunomodulatory effect, which has been extensively studied in human and animal models, using heat- or phenol-killed $P$. acnes suspensions. Its main biological activities include sensitization to the toxic activity of lipopolysaccharide (LPS) [4], activation of macrophages [5], induction of tumoricidal activity [6], increase of the resistance to pathogens $[7,8]$, and adjuvant effect to antibody response [9]. These effects are mediated by proinflammatory cytokines, enhanced by $P$. acnes treatment, such as IFN- $\gamma$, TNF- $\alpha$, IL-1 $\beta$, IL-6, IL-12, and IL-18 [10-14]. The induction of these cytokines by $P$. acnes is dependent on TLR2, TLR9, and MyD88 [15-17]. The bacterium also enhances the expression of TLR2 and TLR4 by keratinocytes [18] and TLR4 and MD-2 by hepatocytes [19], what can explain its ability to potentiate the endotoxic shock to LPS. Recently, it was also demonstrated that $P$. acnes can activate the inflammasome of human peripheral neutrophils, as shown by caspase-1 increased expression [20]. 
Due to the cytokines pattern induced by $P$. acnes, it has been used as a Th1-inducer antigen [21-23]. However, in a previous study from our group, we demonstrated that heatkilled $P$. acnes could not only induce a typical Th1 response, but also enhance the Th2 pattern elicited by another antigen [24], using a murine model of type I hypersensitivity reaction previously described [25].

A soluble polysaccharide component purified from the bacterium cell wall also induced the same responses in such model [26]. This polysaccharide (PS) was purified and characterized by our group [26], and it had already been studied in other models, inducing similar effects to the whole bacterium, such as adjuvant effect to antibody response on a Trypanosoma cruzi DNA vaccine [27], increase of the number and tumoricidal activity of peritoneal macrophages [28], and enhancement of dendritic cells in vivo and in vitro [29]. Recently, PS was also shown to modulate the Th2 response observed in a murine model of focal segmental glomerulosclerosis, inducing a Th1 polarization and kidney preservation [30]. All these results suggest that PS can be a major $P$. acnes component responsible for its effects, including the polarization of $\mathrm{T}$ cell responses.

In the present data, we investigated the mechanisms by which $P$. acnes and PS polarize the immune responses in the type I hypersensitivity model cited above. In such model, described by Facincone et al. [25], F1 BALB/c x $\mathrm{A} / \mathrm{J}$ mice are subcutaneously sensitized with heat-coagulated hen's egg white (HEW) and 14 days later challenged with heat-aggregated OVA in the footpad. A typical late phase reaction (LPR) of immediate hypersensitivity, with intense eosinophilia, is induced [25]. In our previous works using this model, we established two P. acnes- or PS-treatment protocols $[24,26]$. In Protocol 1 (Th2 potentiation), mice were subcutaneously injected with $P$. acnes or PS once a week, during 3 weeks, and HEW was implanted concomitantly to the last P. acnes or PS injection. In Protocol 2 (Th2 suppression), HEW was implanted 1 week after the third dose of $P$. acnes or PS. For each protocol, the respective control group was treated with sterile saline, at the same conditions. Two weeks after HEW implantation, animals were challenged with heat-aggregated OVA in the footpad, as described above. Footpad swelling and histological analysis were performed, determining the number of eosinophils and other inflammatory cells infiltrating the footpad lesions. Intracellular and sera cytokines levels were also analyzed. Protocol 1 increased all the parameters evaluated, indicating Th2 potentiation, while Protocol 2 decreased the response, indicating Th2 suppression $[24,26]$. These data clearly demonstrated that differences between the two protocols occur at the time of HEW implantation, indicating different environment in the moment of antigen capture by antigen-presenting cells (APCs).

Therefore, herein we focused our interest on APCs, which are responsible for $\mathrm{T}$ cell direction. We observed that the activation status of spleen B lymphocytes, macrophages, and dendritic cells was different depending on the treatment protocol with $P$. acnes or PS in the hypersensitivity model. Therefore, the Th1/Th2 polarization by the bacterium or its compound is related to the activation status of APCs, with different patterns of costimulatory molecules and toll-like receptors expression, leading to the production of different types of cytokines.

\section{Material and Methods}

2.1. Animals. Male or female 6-week-old F1 BALB/c x A/J mice were used in all experiments. Animals were housed in standard cages and kept on a 12-hour light/dark cycle, at controlled temperature, with water and food ad libitum. Experimental procedures were approved by the special University Ethics Committee for animal care and experimentation (CEP-1211/2004).

\subsection{Antigens}

Heat-Killed P. acnes Suspension. P. acnes (gently provided by Instituto Adolfo Lutz, SP, Brazil) was cultured in anaerobic medium (Hemobac, Probac, SP, Brazil) for 3 days, at $37^{\circ} \mathrm{C}$. After this period, bacteria were washed three times at 2,000 g, for 30 minutes. The pellet was resuspended in $0.9 \%$ saline and subjected to continuous water vapor for 20 minutes, at $120^{\circ} \mathrm{C}$. The protein concentration of the suspension was determined by the Bradford method [31].

P. acnes Soluble Polysaccharide (PS) Extraction. The polysaccharide extraction and purification were performed as previously described by us [26], based on Palmer and Gerlough protocol [32]. Briefly, $30 \mathrm{~mL}$ of heat-killed P. acnes $(800 \mu \mathrm{g}$ of protein $/ \mathrm{mL}$ ) was mixed with $30 \mathrm{~mL}$ of $90 \%$ phenol and $30 \mathrm{~mL}$ of distilled water and incubated for 10 minutes in $70^{\circ} \mathrm{C}$ water bath. After centrifugation at $2,000 \mathrm{~g}$ and $4^{\circ} \mathrm{C}$, the water phase and the polysaccharide-enriched ring were collected. This step was repeated three times. Three volumes of ethanol were added to each volume of the mixture. After overnight incubation at $4^{\circ} \mathrm{C}$, the precipitate was obtained by centrifugation and designated as the soluble polysaccharide (PS). The carbohydrate concentration was determined by the Dubois method [33].

Heat-Coagulated Hen's Egg White (HEW) for Mice Sensitization. Heat-coagulated HEW was prepared as previously described [25]. The solid egg white was obtained after 30 minutes in boiling water; then, it was isolated, washed in distilled water, and dehydrated in $100 \%$ ethanol overnight. Fragments of approximately $40 \mathrm{mg}$ were obtained and rehydrated in sterile PBS for 2 hours at room temperature, before implantation.

2.3. Type I Hypersensitivity Murine Model. The immediate hypersensitivity model used in our previous studies was developed by Facincone et al. [25]. Accordingly, when F1 $\mathrm{BALB} / \mathrm{c} \times \mathrm{A} / \mathrm{J}$ mice are subcutaneously implanted with heatcoagulated HEW $(40 \mathrm{mg})$ and challenged, after 14 days, with $50 \mu \mathrm{L}$ of heat-aggregated OVA $(20 \mathrm{mg} / \mathrm{mL})$ in the footpad, they develop a late phase reaction of the type I hypersensitivity reaction, characterized by footpad swelling and eosinophil infiltration [25]. In the present work, mice were submitted only to the sensitization phase (HEW implantation) of type I hypersensitivity model. 


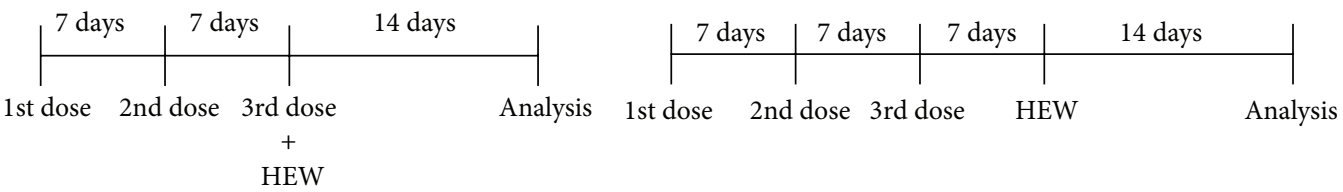

(a) Protocol 1

(b) Protocol 2
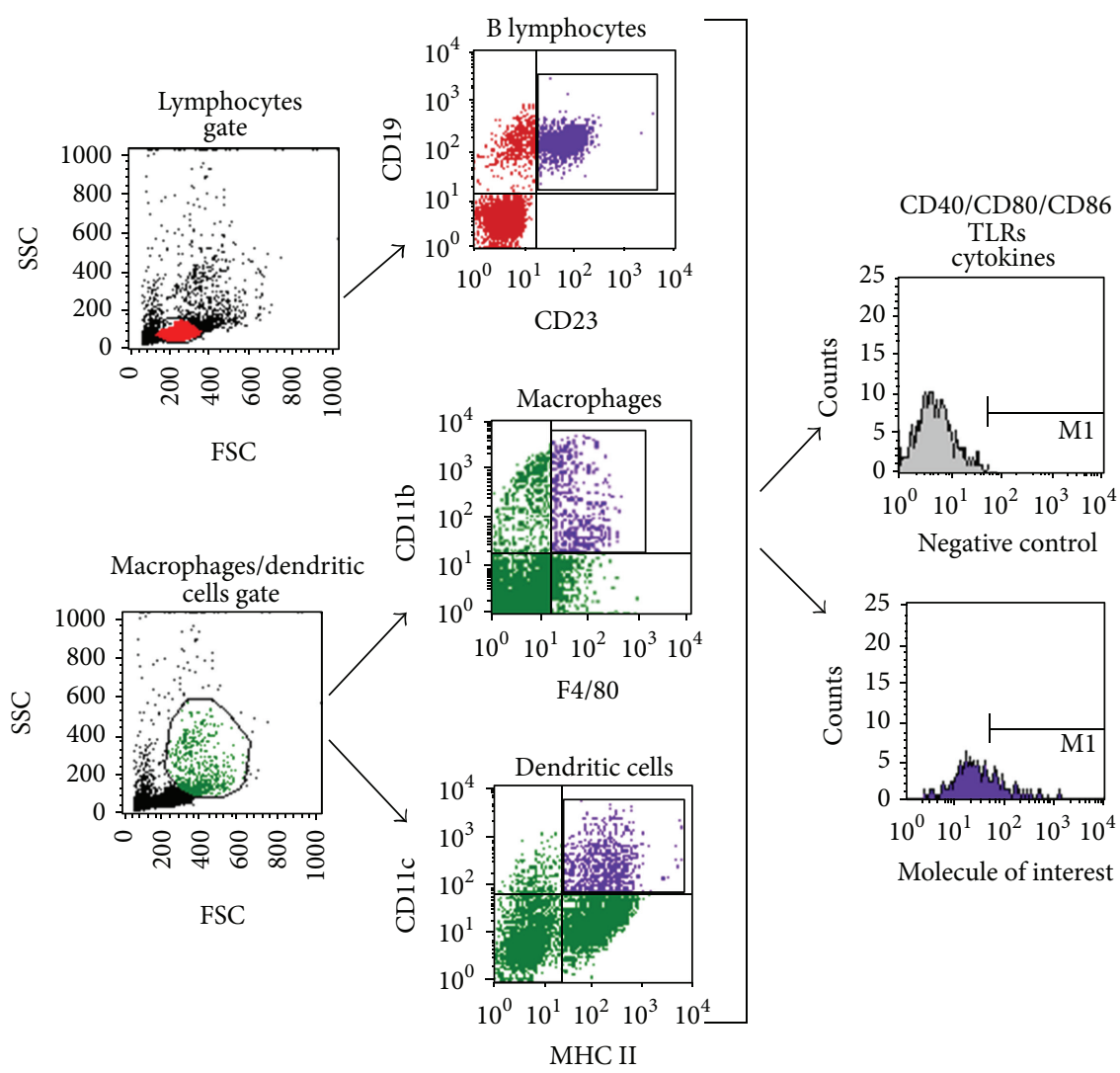

(c) Gates strategy

FIGURE 1: Schematic representation of $P$. acnes- and PS-treatment protocols and gates strategy. F1 BALB/c x A/J mice were subcutaneously injected with $P$. acnes or PS once a week, during 3 weeks. Heat-coagulated hen's egg white (HEW) was implanted concomitantly to the last $P$. acnes or PS dose in Protocol 1 (a) or 1 week after the third dose in Protocol 2 (b). For each protocol, the respective control group was treated with $350 \mu \mathrm{L}$ of sterile commercial $0.9 \%$ saline, at the same conditions. Spleen cells were analyzed 14 days after HEW implantation. (c) Gates strategy used for flow cytometry analysis.

2.4. P. acnes- or PS-Treatment Protocols. As described above, in our previous studies, we established two P. acnes- or PStreatment protocols, which potentiated (Protocol 1) or suppressed (Protocol 2) the Th2 response to OVA after footpad challenge $[24,26]$, and the same protocols were used in the present work. In Protocol 1 (Th2 potentiation), mice were subcutaneously injected with $P$. acnes $(140 \mu \mathrm{g} / 350 \mu \mathrm{L} /$ animal $)$ or PS $(25 \mu \mathrm{g} / 350 \mu \mathrm{L} /$ animal $)$ once a week, during 3 weeks, and HEW was implanted concomitantly to the last P. acnes or PS injection (Figure 1(a)). In Protocol 2 (Th2 suppression), HEW was implanted 1 week after the third dose of $P$. acnes or PS (Figure 1(b)). For each protocol, the respective control group was treated with $350 \mu \mathrm{L}$ of sterile commercial $0.9 \%$ saline, at the same conditions.
Herein, mice were treated according to both protocols described above but not submitted to footpad challenge. Instead, at the moment in which OVA challenge should be done, that is, 14 days after HEW implantation, spleen cells were collected and activation status of APCs was analyzed by flow cytometry (Figure 1).

2.5. Expression of Costimulatory Molecules, TLRs, and Cytokines Synthesis by Spleen Cells. Spleen cells from control, P. acnes- or PS-treated mice were obtained 14 days after HEW implantation. Cellular viability and concentration (total absolute number of cells $/ \mathrm{mL}$ ) were determined by counting with Trypan Blue. Cells $\left(1 \times 10^{6} / 100 \mu \mathrm{L} /\right.$ tube $)$ were then incubated for 30 minutes at $4^{\circ} \mathrm{C}$ with normal mouse serum (5\% in PBS) 
to block Fc receptors. After washing, cells were labeled for 1 hour at $4^{\circ} \mathrm{C}$ with fluorochrome-conjugated monoclonal antimouse antibodies (BD Pharmingen, CA, USA) to determine the expression of costimulatory molecules (CD40, CD80, and CD86), TLRs (TLR2, TLR4, and intra- and extracellular TLR9), and cytokines synthesis (IL-4 and IL-12) by B lymphocytes $\left(\mathrm{CD} 19^{+} \mathrm{CD} 23^{+}\right)$, macrophages $\left(\mathrm{CD}_{11 b^{+}} \mathrm{F} 4 / 80^{+}\right)$, and dendritic cells $\left(\mathrm{CD} 11 \mathrm{c}^{+} \mathrm{MHC} \mathrm{II}^{+}\right)$. TLR9 was analyzed not only intracellularly, but also extracellularly, since the presence of this receptor on the cell membrane of human peripheral blood B lymphocytes and of mouse peritoneal B-1b cells has already been described $[34,35]$.

When intracellular staining had to be performed (intracellular TLR9 and cytokines), cells were washed and fixed (1\% paraformaldehyde) after extracellular labeling and then permeabilized with Triton-X-100 (0.2\% in PBS) for 6 minutes, followed by intracellular labeling for 40 minutes, at room temperature. Cells were then washed and analyzed in a FACSCalibur flow cytometer, using the CellQuest Pro software (Becton Dickinson, CA, USA).

Percentages of positive cells were converted to absolute number (cells $/ \mathrm{mL}$ ) based on the total cell concentration obtained by Trypan Blue counting, once both P. acnes and PS treatments were able to increase the absolute number of total spleen leukocytes (data not shown). The absolute numbers of B lymphocytes, macrophages, and dendritic cells positive for each molecule were added to obtain the total number of spleen APCs positive for such molecule. Expression level of costimulatory molecules, TLRs, and cytokines synthesis was also determined by mean fluorescence intensity (MFI) and individually analyzed in each population. Gates strategy used in flow cytometry analysis is shown in Figure 1(c).

Similarly to what was described above, intracellular staining was performed to determine the absolute number of total spleen cells positive for IL-5 synthesis, an important cytokine in type I hypersensitivity reaction, with an essential role in eosinophil recruitment [36, 37]. Gates strategy used for this analysis is shown in Figure 5(a).

\subsection{Cytokines Detection on Cocultures of \\ Bone Marrow-Derived Dendritic Cells (BMDC) and OVA-Primed T Lymphocytes Stimulated In Vitro with P. acnes, $P S$, and/or OVA}

BMDC Achievement. Femur and tibia from naïve F1 BALB/c $\mathrm{x} \mathrm{A} / \mathrm{J}$ mice were extracted, and their cavities were washed with RPMI (Cultilab, SP, Brazil) to obtain bone marrow cells, which were submitted to $400 \mathrm{~g}$ centrifugation for 5 minutes and resuspended in $1 \mathrm{~mL}$ of R10 (RPMI supplemented with $10 \%$ fetal cow serum). Cellular viability and concentration were determined by counting with Trypan Blue. Based on previously described protocols to obtain BMDC [21-23], cells $\left(2 \times 10^{5} / \mathrm{mL}\right)$ were cultured for 10 days at $37^{\circ} \mathrm{C}$ and $5 \% \mathrm{CO}_{2}$ in R10 supplemented with GM-CSF $(20 \mathrm{ng} / \mathrm{mL})$, replacing the medium on days 3,6 , and 8 . At 10th day, GMCSF concentration was reduced to $5 \mathrm{ng} / \mathrm{mL}$; OVA-primed $\mathrm{T}$ lymphocytes and in vitro stimuli were then added, as described below.
OVA-Primed T Lymphocytes Enrichment. Naïve F1 BALB/c $\mathrm{x} \mathrm{A} / \mathrm{J}$ mice were submitted to HEW implantation, and after 14 days, spleen cells were obtained and mixed to carbonyl iron $(0.1 \mathrm{mg} / \mathrm{mL})$ to increase macrophage density. After incubation at $37^{\circ} \mathrm{C}$ for 30 minutes, macrophage-depleted supernatant cells were collected, washed, and submitted to Histopaque (Sigma-Aldrich, MO, USA) density gradient centrifugation $(d=1.077 \mathrm{~g} / \mathrm{mL})$, for 30 minutes, at $400 \mathrm{~g}$. Mononuclear cells-enriched ring was collected and washed with RPMI, for 5 minutes, at $400 \mathrm{~g}$. Cell suspension was then transferred twice to Petri dishes, previously coated with purified anti-mouse IgM $(10 \mu \mathrm{g} / \mathrm{mL})$, and incubated for 1 hour at $37^{\circ} \mathrm{C}$ and $5 \% \mathrm{CO}_{2}$, for $\mathrm{B}$ cell depletion. Supernatant cells were collected, and purity was verified by flow cytometry. A T cell-enriched population (95\%) was obtained and added to BMDC cultures, as described below.

BMDC and OVA-Primed T Lymphocytes Cocultures. At 10th day of BMDC culture, OVA-primed T lymphocytes $(5 \times$ $10^{5} / \mathrm{mL}$ ) were added, and different in vitro stimuli were performed as follows: (a) cultures stimulated or not with P. acnes $(2.5 \mu \mathrm{g} / \mathrm{mL})$, PS $(0.5 \mu \mathrm{g} / \mathrm{mL})$, or OVA $(17.5 \mu \mathrm{g} / \mathrm{mL})$ at 10th day; (b) cultures stimulated with $P$. acnes or PS, concomitantly to OVA, at 10th day; (c) cultures stimulated with $P$. acnes or PS at 10th day and OVA at 11th day. All supernatants were collected at 12 th day and submitted to ELISA for IL-4, IL-5, IL-12, IL-17, and IFN- $\gamma$ detection.

ELISA for Cytokines Detection. IL-17 detection was performed according to the manufacturer's instructions (eBioscience, CA, USA). For the other cytokines, plates were coated with $50 \mu \mathrm{L} /$ well of purified monoclonal anti-mouse IL-4 $(1 \mu \mathrm{g} / \mathrm{mL})$, anti-mouse IL-5 $(2 \mu \mathrm{g} / \mathrm{mL})$, anti-mouse IL-12 $(4 \mu \mathrm{g} / \mathrm{mL})$, or anti-mouse IFN $-\gamma(6 \mu \mathrm{g} / \mathrm{mL})$ (R\&D Systems, MN, USA) overnight, at room temperature, and washed three times with PBS-Tween $200.05 \%$ (200 $\mu \mathrm{L} /$ well). After blocking free sites with PBS-BSA $1 \%(200 \mu \mathrm{L} /$ well $)$, for 1 hour, at room temperature, plates were washed again, and recombinant cytokines $(15 \mathrm{pg} / \mathrm{mL}$ to $4,000 \mathrm{pg} / \mathrm{mL}$ ) (R\&D Systems) or samples $(50 \mu \mathrm{L} /$ well) were incubated for 2 hours at room temperature. After washing, biotinylated monoclonal antimouse IL-4 (400 ng/mL), anti-mouse IL-5 (400 ng/mL), antimouse IL-12 $(200 \mathrm{ng} / \mathrm{mL})$, or anti-mouse IFN- $\gamma(400 \mathrm{ng} / \mathrm{mL})$ (R\&D Systems) was added ( $50 \mu \mathrm{L} /$ well) and incubated for 2 hours, at room temperature. Plates were washed again and incubated with $50 \mu \mathrm{L} /$ well of HRP-conjugated streptavidin $(1: 4,000)$ (BD Pharmingen) for 30 minutes, at room temperature. After washing, peroxidase activity was assessed with OPD-substrate and stopped with $4 \mathrm{~N}_{2} \mathrm{SO}_{4}$. Optical density was determined at $492 \mathrm{~nm}$. Cytokines levels were calculated based on recombinant cytokines curves.

2.7. Statistical Analysis. The statistical differences between control and treated groups were analyzed by one-way ANOVA followed by Tukey's multiple comparison posttest, using GraphPad Prism software (GraphPad Software, CA, USA). Differences were taken as statistically significant when $P<0.05$. 


\section{Results}

3.1. P. acnes or PS Treatments according to Protocol 1 (Th2 Potentiation) Increased the Absolute Number and Expression Level of $\mathrm{CD}^{4} 0^{+}, \mathrm{CD}^{\circ} 0^{+}$, and $\mathrm{CD}^{+} 6^{+}$Spleen APCs. Absolute numbers of spleen $B$ lymphocytes, macrophages, and dendritic cells $\mathrm{CD} 40^{+}, \mathrm{CD} 80^{+}$, or $\mathrm{CD} 86^{+}$, from each treated group, were added to obtain the total number of spleen APCs positive for these costimulatory molecules, while expression level (MFI) was analyzed individually for each population.

We observed that $P$. acnes and PS treatments according to Protocol 1 increased the absolute number of APCs expressing costimulatory molecules, except CD40 in PS group (Figure 2(a)), and also enhanced their expression levels by macrophages and dendritic cells (Figures 2(c) and 2(d)). PS also upregulated the expression of CD80 and CD86 by B lymphocytes (Figure 2(b)).

3.2. In General, P. acnes or PS Treatments according to Protocol 2 (Th2 Suppression) Slightly Increased or Even Decreased the Absolute Number of Costimulatory Positive Spleen APCs and Reduced Their Expression Levels. P. acnes treatment according to Protocol 2 enhanced the number of APCs expressing CD80 and CD86, but at a much lower level than Protocol 1, besides having impaired the number of $\mathrm{CD} 40^{+}$cells (Figure 2(e)). PS decreased the number of cells expressing all three analyzed molecules (Figure 2(e)). In addition, there was also a decrease in the expression level of CD80 and CD86 by B lymphocytes from $P$. acnes-treated group (Figure 2(f)), as well as CD40 by macrophages (Figure $2(\mathrm{~g})$ ), and only a slight increase of CD80 by dendritic cells (Figure 2(h)). PS downregulated the expression of the three molecules by macrophages (Figure 2(g)), CD40 and CD86 by dendritic cells (Figure 2(h)), and CD86 by B lymphocytes (Figure 2(f)).

3.3. Both Protocols Increased the Number of APCs Expressing TLRs, but the Expression Levels of These Molecules Were Predominantly Enhanced in Mice Submitted to Protocol 2 (Th2 Suppression), Mainly in P. acnes Group. In mice submitted to Protocol 1, there was an increase in the absolute number of spleen APCs expressing TLR2, TLR4, and extra- and intracellular TLR9 in P. acnes and PS groups, except intracellular TLR9 for PS group (Figure 3(a)). P. acnes treatment increased the expression level of intracellular TLR9 by B lymphocytes and macrophages, while PS increased extracellular TLR9 in these populations (Figures 3(b) and 3(c)). PS also upregulated the expression of TLR2 and intracellular TLR9 by macrophages (Figure 3(c)). On the other hand, P. acnes decreased the expression of TLR2 by B lymphocytes (Figure 3(b)), TLR4 by macrophages (Figure 3(c)), and extra- and intracellular TLR9 by dendritic cells (Figure 3(d)). PS only decreased the expression of intracellular TLR9 by dendritic cells (Figure 3(d)).

In Protocol 2, there was also an increase in the absolute number of spleen APCs expressing TLR2 and extraand intracellular TLR9 in P. acnes and PS groups, but not TLR4 (Figure 3(e)). Differently from Protocol 1, $P$. acnes upregulated the expression of all TLRs by B lymphocytes (Figure 3(f)), extracellular TLR9 by macrophages
(Figure 3(g)), and TLR2, TLR4, and intracellular TLR9 by dendritic cells (Figure 3(h)). PS also increased the expression of extracellular TLR9 by B lymphocytes (Figure 3(f)), TLR2, TLR4, and extracellular TLR9 by macrophages (Figure 3(g)), and intracellular TLR9 by dendritic cells (Figure 3(h)).

3.4. P. acnes and PS Treatments according to Protocol 1 (Th2 Potentiation) Increased $\mathrm{IL}-4^{+}$and Decreased $\mathrm{IL}-12^{+}$Spleen APCs Absolute Number. Confirming the potentiation of the Th2 response to OVA, an increase in the number of spleen APCs synthesizing IL-4 in mice treated with $P$. acnes or PS according to Protocol 1 was observed, with a concomitant decrease in the number of IL- $12^{+}$cells (Figure 4(a)). Interestingly, $P$. acnes induced lower levels of these two cytokines on B lymphocytes and dendritic cells (Figures 4(b) and 4(d)) but increased the production of IL-12 by macrophages (Figure $4(\mathrm{c})$ ). On the other hand, PS increased the production of IL4 and IL-12 by macrophages and dendritic cells (Figures 4(c) and $4(\mathrm{~d}))$.

3.5. P. acnes and PS Treatments according to Protocol 2 (Th2 Suppression) Increased Both $\mathrm{IL}-4^{+}$and $\mathrm{IL}-12^{+}$Spleen APCs Absolute Number, but IL-4 $4^{+}$Cells Increment Was Not so Pronounced as in Protocol 1 (Th2 Potentiation). In Protocol 2 , both treatments also elevated the number of APCs synthesizing IL-4, but this increase was not so marked as the one observed in Protocol 1 (Figure 4(e)). Besides, differently from Protocol 1, P. acnes and PS treatments according to Protocol 2 induced an increase in the number of APCs positive for IL-12 (Figure 4(e)), confirming the suppression of Th2 response. $P$. acnes treatment also increased the production of IL-12 by B lymphocytes (Figure 4(f)) and decreased IL-4 by macrophages and dendritic cells (Figures $4(\mathrm{~g})$ and $4(\mathrm{~h})$ ), while PS decreased the synthesis of IL- 4 by B cells (Figure $4(\mathrm{f}))$.

3.6. The Numbers of $I L-5^{+}$Spleen Cells Confirmed the Th2 Potentiation in Protocol 1 and the Suppression in Protocol 2 by Both P. acnes and PS Treatments. P. acnes and PS treatments according to Protocol 1 also increased the number of total spleen cells positive for IL-5, unlike the one observed in Protocol 2, in which $P$. acnes and PS treatments decreased the number of IL- $5^{+}$cells (Figure 5(b)). These data also confirm Th2 potentiation response in Protocol 1 and Th2 suppression in Protocol 2.

3.7. Higher Levels of IL-5 and IL-17 Were Detected in Cocultures of Bone Marrow-Derived Dendritic Cells (BMDC) and OVAPrimed T Lymphocytes Submitted to Protocol 1-Like (Th2 potentiation), Compared to Lower Levels in Protocol 2-Like (Th2 Suppression). In order to confirm the direct effect of $P$. acnes and PS on antigen-presenting cells in this model of type I hypersensitivity reaction, we performed a coculture of BMDC and OVA-primed T lymphocytes, differently stimulated with $P$. acnes, PS, and/or OVA, analyzing the pattern of cytokines released in the culture supernatant.

BMDC were obtained after culturing bone marrow cells for 10 days with GM-CSF. At 10th culture day of BMDC, 
Protocol 1

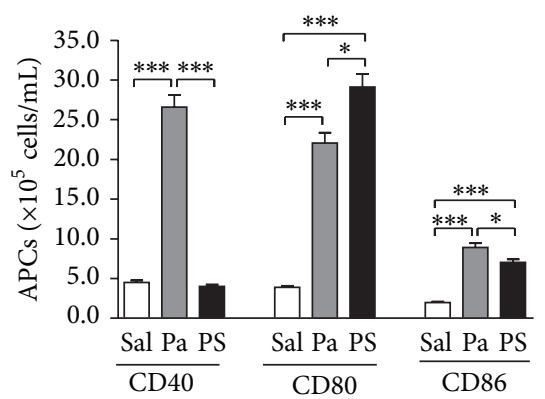

(a)

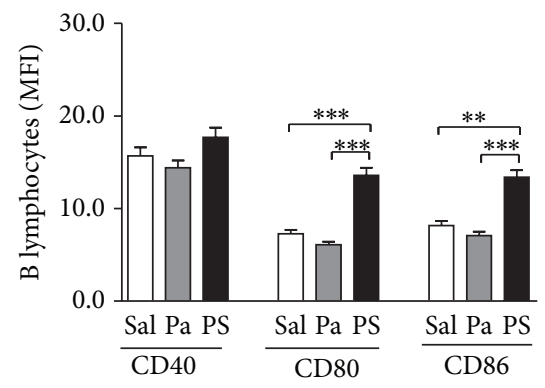

(b)

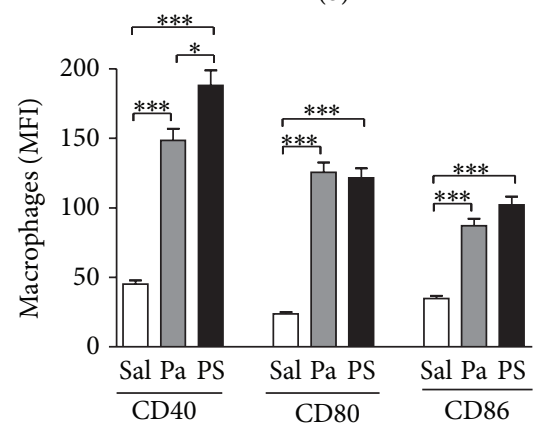

(c)

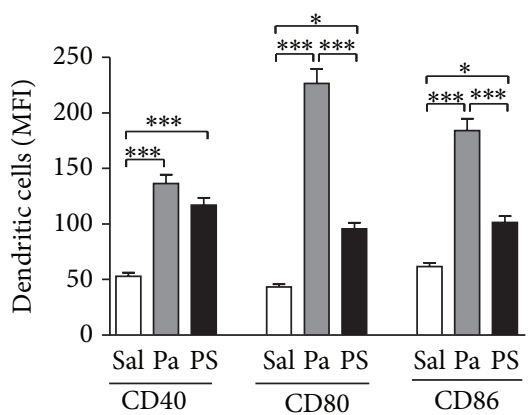

(d)
Protocol 2

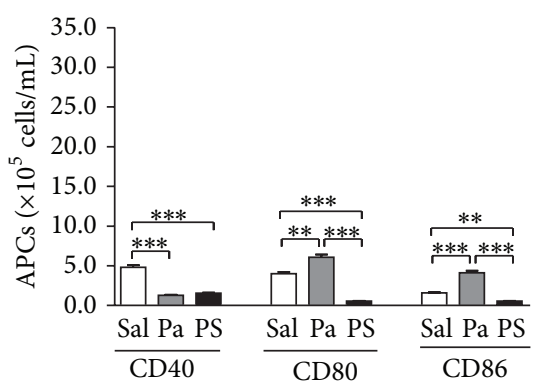

(e)

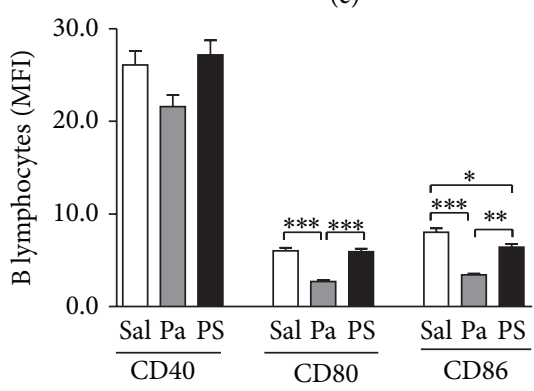

(f)

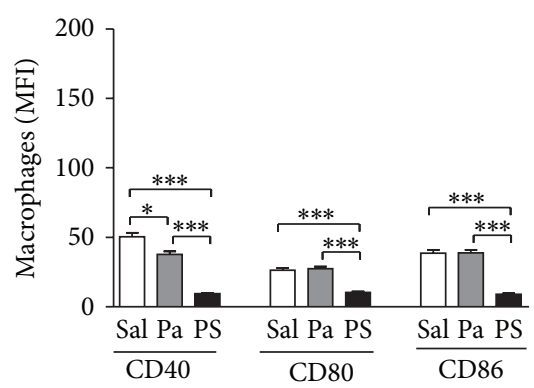

(g)

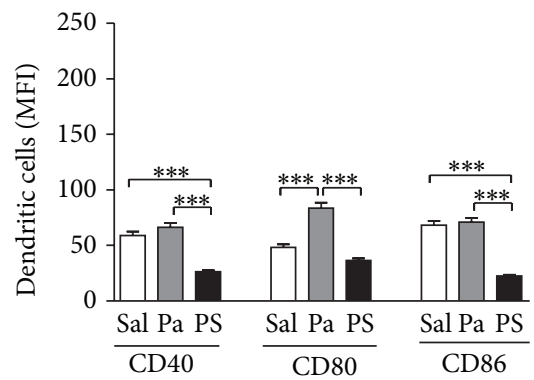

(h)

FIGURE 2: Expression of costimulatory molecules by spleen antigen-presenting cells. Spleen cells from mice treated with saline (Sal), P. acnes (Pa), or PS were obtained 14 days after HEW implantation and stained with fluorochrome-conjugated monoclonal anti-mouse antibodies to determine the expression of CD40, CD80, and CD86 by B lymphocytes, macrophages, and dendritic cells. Percentages obtained by flow cytometric analysis were converted to absolute numbers of B lymphocytes, macrophages, and dendritic cells positive for each molecule, which were added to obtain the total number of spleen APCs positive for such molecule in Protocols 1 (a) and 2 (e). Expression levels were also determined by mean fluorescence intensity (MFI) and individually analyzed in B lymphocytes ((b) and (f)), macrophages ((c) and (g)), and dendritic cells ((d) and (h)) for Protocols 1 and 2, respectively. ${ }^{*} P<0.05 ;{ }^{* *} P<0.01 ;{ }^{* * *} P<0.0001$. 
Protocol 1

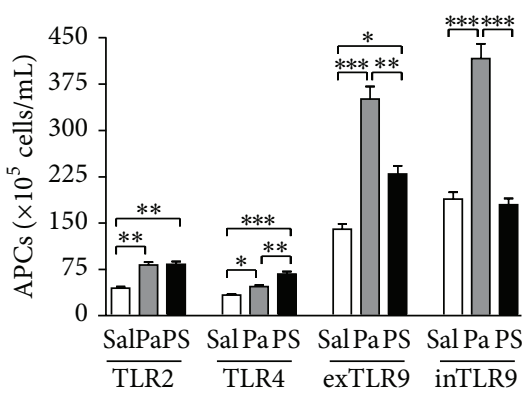

(a)

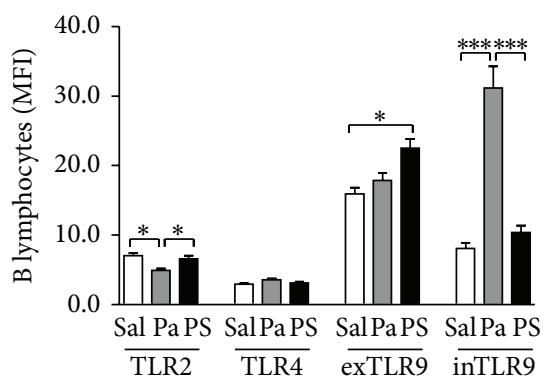

(b)

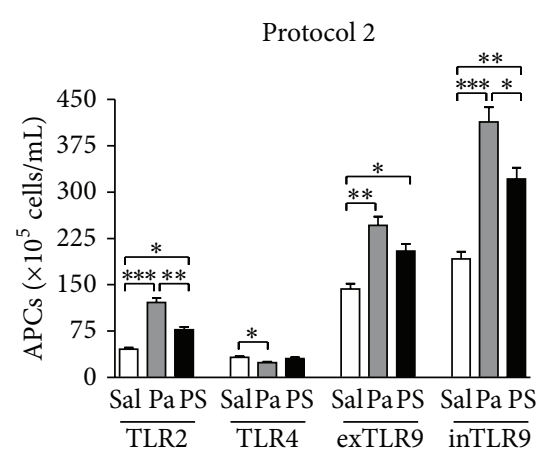

(e)

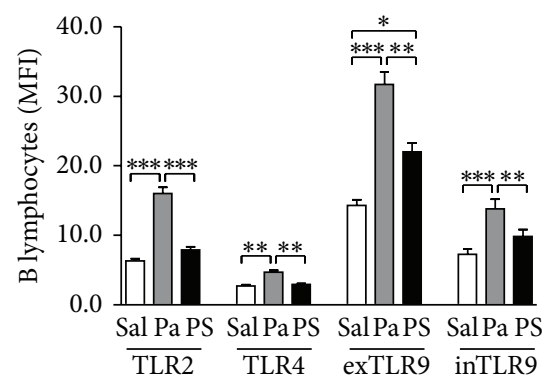

(f)

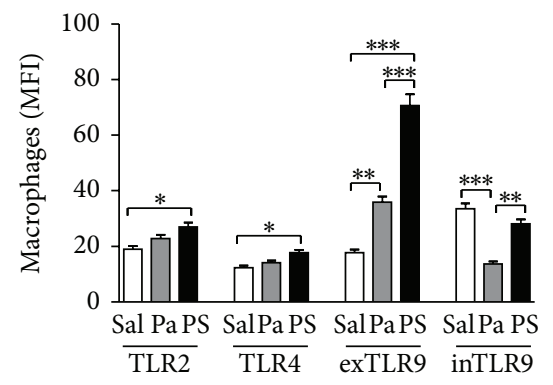

(g)

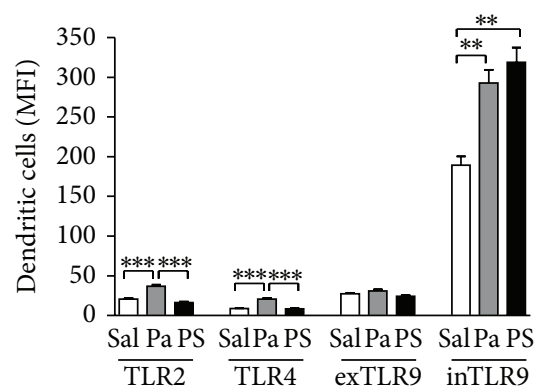

(h)

FIgURE 3: Expression of toll-like receptors by spleen antigen-presenting cells. Spleen cells from mice treated with saline (Sal), P. acnes (Pa), or PS were obtained 14 days after HEW implantation and stained with fluorochrome-conjugated monoclonal anti-mouse antibodies to determine the expression of TLR2, TLR4, and intra- and extracellular TLR9 by B lymphocytes, macrophages, and dendritic cells. Percentages obtained by flow cytometric analysis were converted to absolute numbers of B lymphocytes, macrophages, and dendritic cells positive for each receptor, which were added to obtain the total number of spleen APCs positive for such receptor in Protocols 1 (a) and 2 (e). Expression levels were also determined by mean fluorescence intensity (MFI) and individually analyzed in B lymphocytes ((b) and (f)), macrophages ((c) and (g)), and dendritic cells ((d) and (h)) for Protocols 1 and 2 , respectively. ${ }^{*} P<0.05 ;{ }^{* *} P<0.01 ;{ }^{* * *} P<0.0001$. 


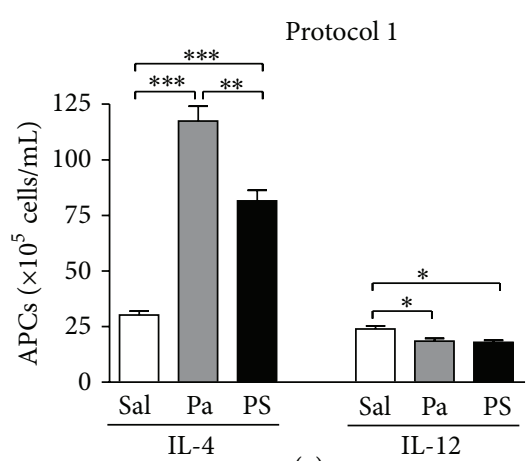

(a)

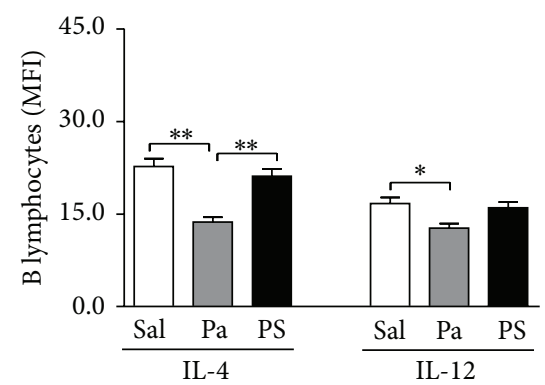

(b)

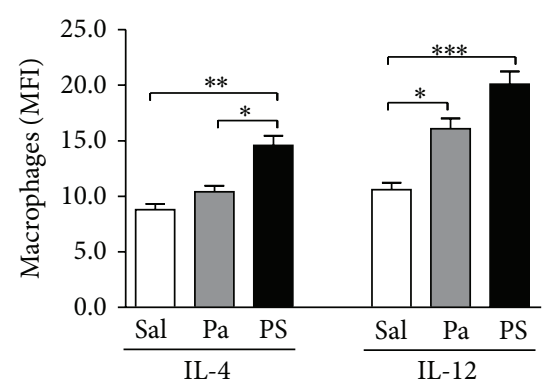

(c)

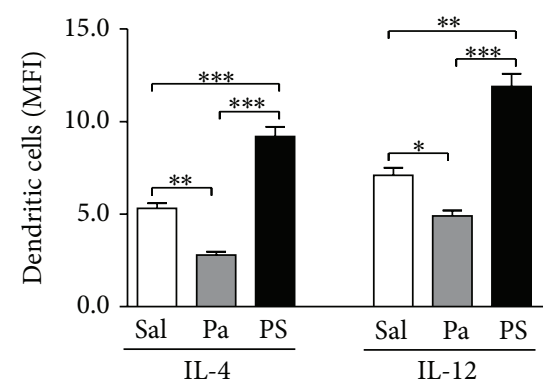

(d)
Protocol 2

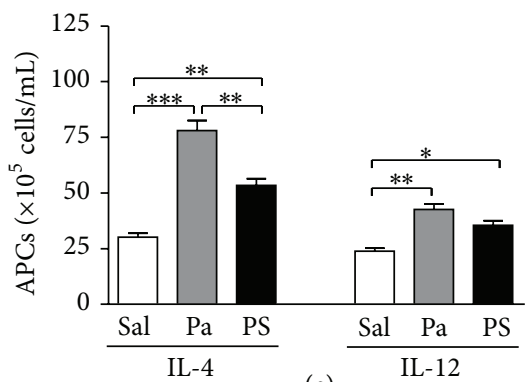

(e)

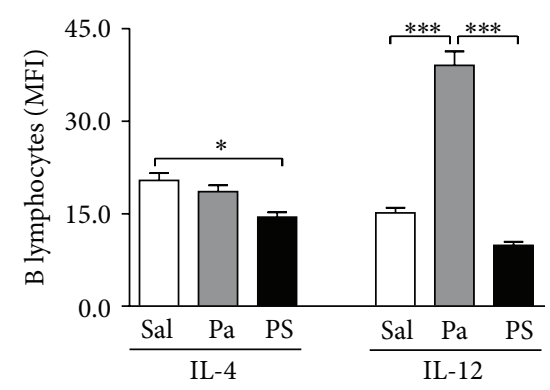

(f)

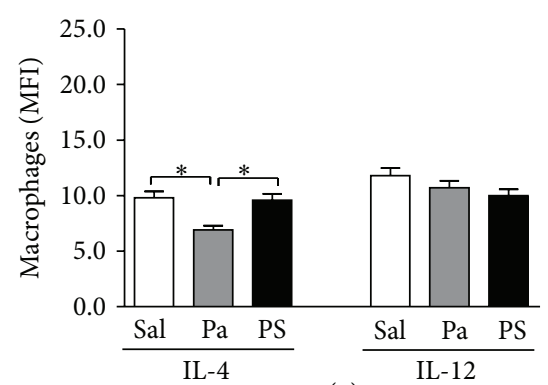

(g)

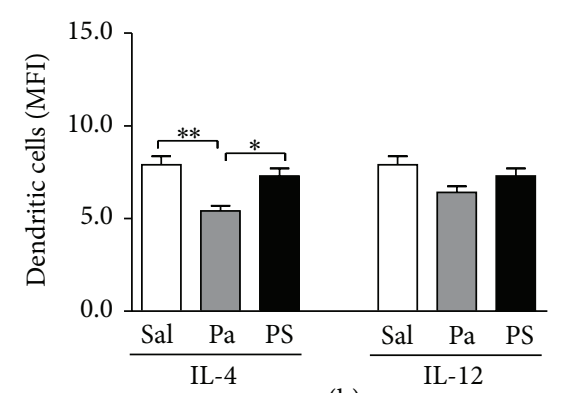

(h)

FIGURE 4: Cytokines synthesis by spleen antigen-presenting cells. Spleen cells from mice treated with saline (Sal), P. acnes (Pa), or PS were obtained 14 days after HEW implantation and stained with fluorochrome-conjugated monoclonal anti-mouse antibodies to determine the intracellular synthesis of IL- 4 and IL-12 by B lymphocytes, macrophages, and dendritic cells. Percentages obtained by flow cytometric analysis were converted to absolute numbers of B lymphocytes, macrophages, and dendritic cells positive for each cytokine, which were added to obtain the total number of spleen APCs positive for such cytokine in Protocols 1 (a) and 2 (e). Production levels were also determined by mean fluorescence intensity (MFI) and individually analyzed in B lymphocytes ((b) and (f)), macrophages ((c) and (g)), and dendritic cells ((d) and (h)) for Protocols 1 and 2 , respectively. ${ }^{*} P<0.05$; $^{* *} P<0.01$; $^{* * *} P<0.0001$.

OVA-primed T lymphocytes were added. To reproduce the potentiation protocol (Protocol 1-like), cocultures were stimulated with P. acnes and OVA or PS and OVA at 10th day, and supernatants were analyzed at 12 th day. To reproduce the suppression protocol (Protocol 2-like), cocultures were stimulated with P. acnes or PS at 10th day and with OVA at 11th day and supernatants were analyzed at 12th day.

IL-4, IL-12, and IFN- $\gamma$ were not detected in any culture (data not shown). In vitro stimulus with OVA alone (antigenspecific positive control) induced the release of detectable 


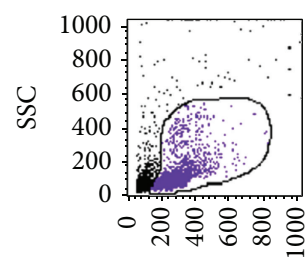

FSC

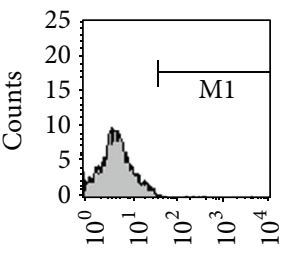

Negative control

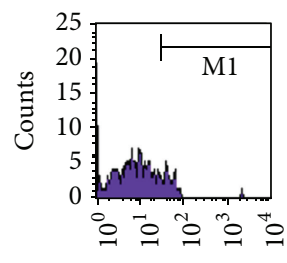

IL-5

(a)

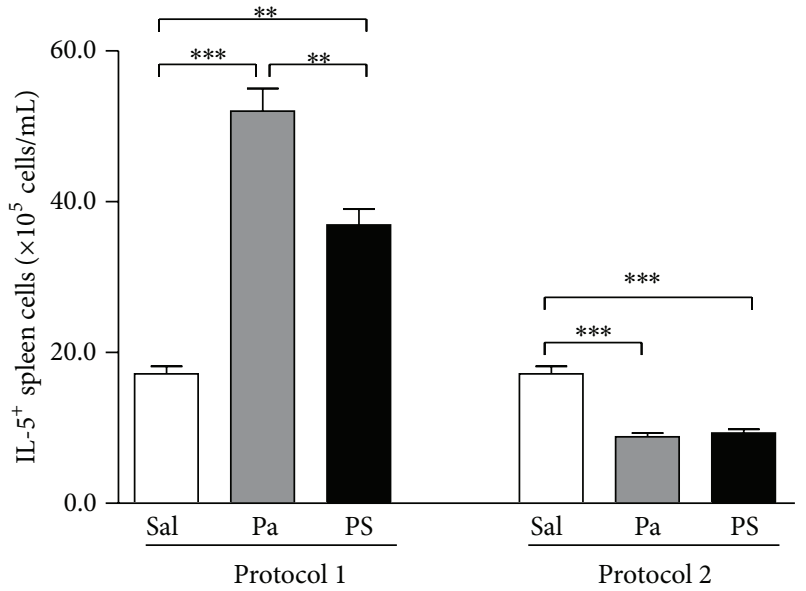

(b)

FIGURE 5: IL-5 synthesis by spleen cells. Spleen cells from mice treated with saline (Sal), P. acnes (Pa), or PS were obtained 14 days after HEW implantation and intracellularly stained with fluorochrome-conjugated monoclonal anti-IL-5 antibody. Percentages obtained by flow cytometric analysis (a) were converted to absolute numbers of IL $-5^{+}$spleen cells (b). ${ }^{* *} P<0.01 ;{ }^{* * *} P<0.0001$.

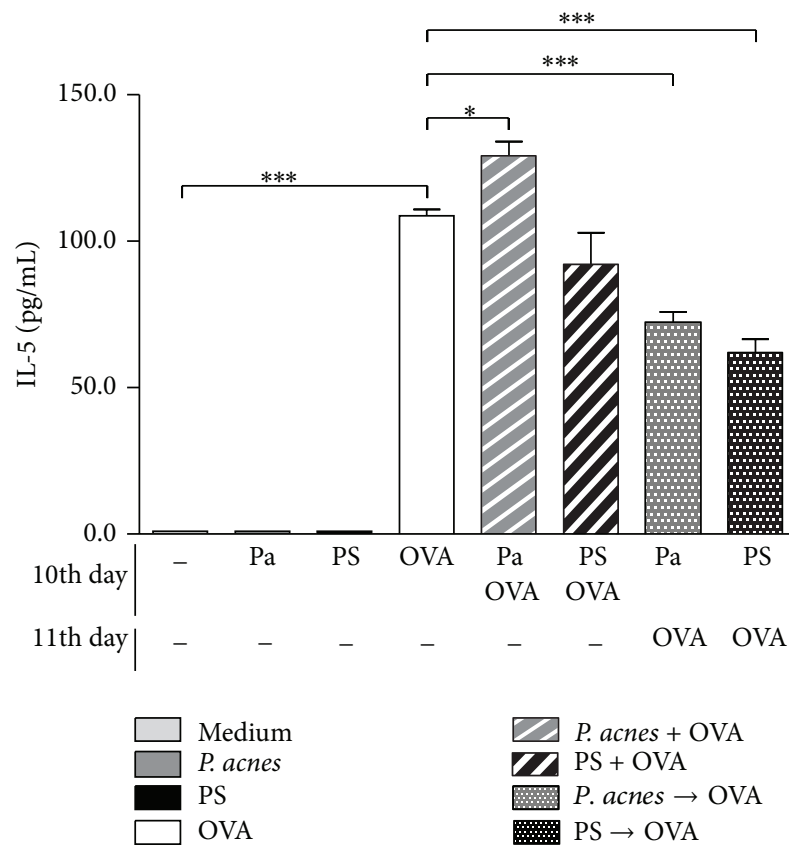

(a)
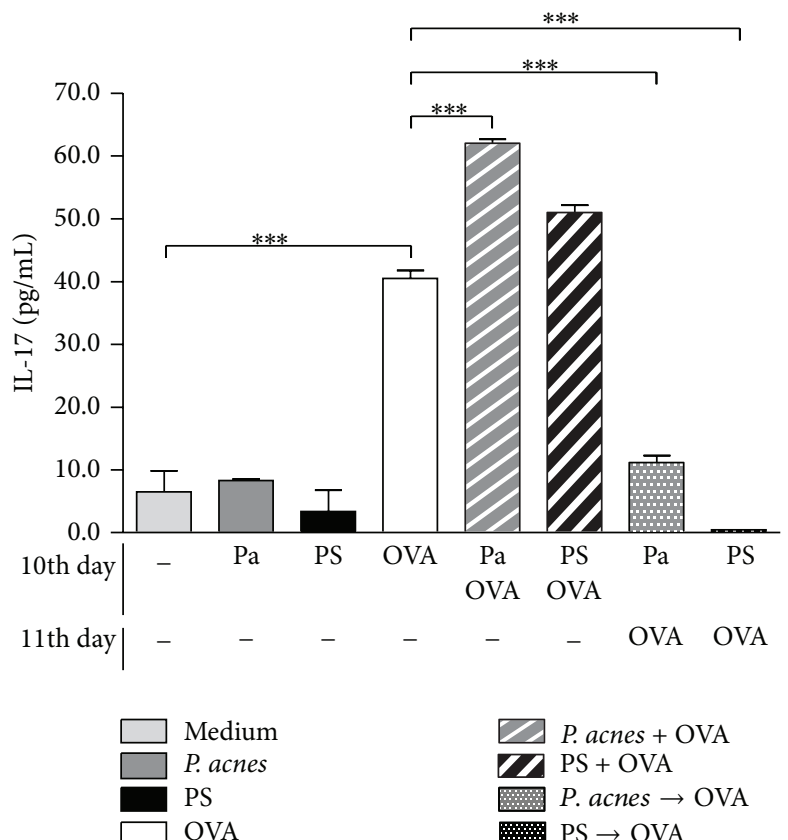

OVA

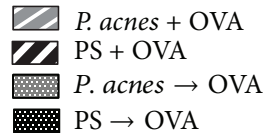

(b)

FIGURE 6: Cytokines production in cocultures of bone marrow-derived dendritic cells and OVA-primed T lymphocytes. Bone marrow cells from naïve $\mathrm{F} 1 \mathrm{BALB} / \mathrm{c} \times \mathrm{A} / \mathrm{J}$ mice were cultured during 10 days with GM-CSF, for dendritic cell differentiation. At 10th day, enriched OVAprimed T lymphocytes, obtained from the spleen of HEW-implanted F1 BALB/c x A/J mice, were added to the cultures. The following in vitro stimuli were performed: $P$. acnes, PS, or OVA at 10th day; P. acnes or PS, concomitantly to OVA, at 10th day (P. acnes + OVA and PS + OVA, resp.); P. acnes or PS at 10th day and OVA at 11th day (P. acnes $\rightarrow$ OVA and PS $\rightarrow$ OVA, resp.). Supernatants were collected at 12 th day and submitted to ELISA for IL-5 (a) and IL-17 (b) detection. ${ }^{*} P<0.05$; ${ }^{* * *} P<0.0001$.

levels of IL-5, when compared to the negative controls (medium, P. acnes or PS) (Figure 6(a)). There was a significant increase in these levels when cocultures were submitted to Protocol 1-like ( $P$. acnes + OVA), but a significant decrease when using the Protocol 2-like $(P$. acnes $\rightarrow$ OVA or PS $\rightarrow$ OVA) (Figure 6(a)). Similar results were observed for the production of IL-17, whose levels were elevated in cultures submitted to the potentiation protocol (P. acnes + OVA) and drastically impaired in the suppression protocol ( $P$. acnes $\rightarrow$ OVA or PS $\rightarrow$ OVA) (Figure 6(b)). These data suggest that $P$. acnes or PS can directly act on antigen-presenting cells to modulate the Th2 response to OVA. 


\section{Discussion}

In previous studies from our group, we demonstrated that, depending on the treatment protocol, $P$. acnes or PS could suppress or enhance the Th2 response to OVA in a murine model of type I hypersensitivity reaction $[24,26]$. Herein, we investigated the mechanisms of action of $P$. acnes and PS in Th1/Th2 polarization, focusing on the immunophenotype and the activation status of APCs, which are responsible for driving the $\mathrm{T}$ helper response.

As previously described, Protocols 1 (Th2 potentiation) and 2 (Th2 suppression) differ only at the time of HEW implantation $[24,26]$. Therefore, we hypothesized that $P$. acnes and PS could modulate the activation status of APCs during sensitization phase, leading to different responses to OVA challenge. In order to confirm this hypothesis, spleen cells from mice submitted to Protocols 1 (Th2 potentiation) and 2 (Th2 suppression) were analyzed at the moment in which OVA challenge should be done, that is, 14 days after HEW implantation (Figures 1(a) and 1(b)).

The activation status of spleen B lymphocytes, macrophages, and dendritic cells was analyzed by the expression of costimulatory molecules, TLRs, and cytokines production, considering both the number of positive cells and the expression level of each molecule analyzed.

According to our and others' previous publications, the better way to represent the modulatory effects of $P$. acnes and PS is analyzing the absolute number of cells, rather than the percentage, once both treatments are able to increase the total number of leukocytes in different organs, according to the inoculation route. In fact, among other organs, $P$. acnes increases the number of leukocytes in spleen, liver, and peritoneal exudate $[13,16,28]$.

A great increase in the number of APCs expressing costimulatory molecules in P. acnes and PS groups submitted to Protocol 1 (Th2 potentiation) was observed, except CD40 in PS group (Figure 2(a)), as well as upregulation of the three molecules, mainly by macrophages and dendritic cells (Figures 2(b) to 2(d)). On the other hand, in Protocol 2 (Th2 suppression), there was little increase or even decrease in the number of positive APCs (Figure 2(e)), as well as downregulation of CD40, CD80, and CD86 (Figures 2(f) to 2(h)). Table 1(a) summarizes the effects of $P$. acnes and PS on the expression levels of costimulatory molecules and clearly shows that both treatments mainly upregulate these molecules in Protocol 1 and downregulate in Protocol 2.

These results suggest that higher number of positive APCs and higher expression levels of costimulatory molecules could be related to potentiation of Th2 response to OVA by $P$. acnes and PS. In fact, several studies have demonstrated the role of costimulatory molecules in different models of Th2 responses. Lindell et al. (2008) verified that B cells from allergen-challenged mice upregulated the expression of CD40, CD80, and CD86 and induced the production of Th2 cytokines by $\mathrm{T}$ cells [38]. It has also been demonstrated that CD80 and CD86 are critical and potent stimulators of Th2 differentiation in vitro [39] and that these molecules are essential for the generation of Th2 cells during the sensitization phase in a murine model of asthma [40]. Similarly, it has
TABLE 1: Schematic representation of $P$. acnes and PS effects on costimulatory molecules and TLRs expression in Protocols 1 and 2. P. acnes and PS effects on co-stimulatory molecules (a) and TLRs (b) expression in Protocols 1 and 2 are summarized and represented as different symbols. $(\bullet)$ indicates up-regulation, while $(O)$ indicates down-regulation of each molecule compared to the control group. $(-)$ indicates that there was no difference in the expression of the molecule in relation to the control group.

(a)

\begin{tabular}{|c|c|c|c|c|c|c|}
\hline \multirow{2}{*}{ P. acnes } & \multicolumn{3}{|c|}{ Protocol 1} & \multicolumn{3}{|c|}{ Protocol 2} \\
\hline & $\mathrm{B}$ & M & $\mathrm{DC}$ & B & M & DC \\
\hline CD40 & - & - & $\bullet$ & - & 0 & - \\
\hline CD80 & - & $\bullet$ & - & O & - & - \\
\hline CD86 & - & $\bullet$ & $\bullet$ & 0 & - & - \\
\hline \multirow{2}{*}{ PS } & \multicolumn{3}{|c|}{ Protocol 1} & \multicolumn{3}{|c|}{ Protocol 2} \\
\hline & $\mathrm{B}$ & M & $\mathrm{DC}$ & B & M & DC \\
\hline CD40 & - & 0 & $\bullet$ & - & 0 & 0 \\
\hline CD80 & $\bullet$ & $\bullet$ & - & - & 0 & - \\
\hline CD86 & $\bullet$ & 0 & • & 0 & 0 & 0 \\
\hline
\end{tabular}

(b)

\begin{tabular}{|c|c|c|c|c|c|c|}
\hline \multirow{2}{*}{ P. acnes } & \multicolumn{3}{|c|}{ Protocol 1} & \multicolumn{3}{|c|}{ Protocol 2} \\
\hline & B & M & $\mathrm{DC}$ & B & M & $\mathrm{DC}$ \\
\hline TLR2 & $\bigcirc$ & - & - & 0 & - & ○ \\
\hline TLR4 & - & $\bigcirc$ & - & 0 & - & $\bullet$ \\
\hline exTLR9 & - & - & $\mathrm{O}$ & 0 & O & - \\
\hline inTLR9 & 0 & 0 & $\mathrm{O}$ & 0 & $\mathrm{O}$ & 0 \\
\hline \multirow{2}{*}{ PS } & \multicolumn{3}{|c|}{ Protocol 1} & \multicolumn{3}{|c|}{ Protocol 2} \\
\hline & $\mathrm{B}$ & M & $\mathrm{DC}$ & $\mathrm{B}$ & $\mathrm{M}$ & $\mathrm{DC}$ \\
\hline TLR2 & - & 0 & - & - & 0 & - \\
\hline TLR4 & - & - & - & - & ○ & - \\
\hline exTLR9 & 0 & 0 & - & 0 & 0 & - \\
\hline inTLR9 & 一 & 0 & $\mathrm{O}$ & 一 & 一 & 0 \\
\hline
\end{tabular}

been shown that the expression of CD40 by dendritic cells is essential for the initiation of Th2 responses [22]. Moreover, several data revised by Lenschow et al. (1996) indicate that higher stimulation of $\mathrm{CD} 28$ by $\mathrm{B} 7$ molecules is related to the induction of Th2 responses [41].

On the other hand, we hypothesize that the downregulation of costimulatory molecules observed in Protocol 2 can be related to Th2 suppression. It is important to note that the downregulation of costimulatory molecules does not mean that APCs are less activated, because other parameters, such as TLRs expression and cytokines production, have to be considered in order to determine the activation status of these cells.

Concerning the effects of PS on the modulation of CD40, it is important to note that the modulation of the number of $\mathrm{CD} 40^{+}$cells is not necessarily related to the modulation of the CD40 levels in cell membrane, and the same applies to all the other molecules studied. Besides, differences observed between $P$. acnes and PS treatments can be explained by the presence of other compounds in the whole bacterium, such as proteins and lipids, which could be responsible for the 
increase in the $\mathrm{CD} 40^{+}$population in Protocol 1, explaining why the same effect is not observed in PS group.

$P$. acnes and PS also modulated the number of APCs expressing TLRs, but the differences between Protocols 1 (Th2 potentiation) and 2 (Th2 suppression) were not as pronounced as that observed for costimulatory molecules, except for TLR4 ${ }^{+}$cells, which were increased in Protocol 1 (Figure 3(a)) and decreased in Protocol 2 (Figure 3(e)). On the other hand, as shown in Figure 3 and summarized in Table 1(b), the expression level of TLRs was enhanced in Protocol 2 (Th2 suppression) rather than Protocol 1 (Th2 potentiation), mainly in $P$. acnes-treated groups. These data suggest that higher expression of these receptors could be related to the suppression of Th2 responses. Indeed, several studies have shown the relationship between TLR signaling and the induction of Th1 responses or suppression of Th2 pattern in allergic diseases. In farmers' families, LPS exposure and higher levels of TLR2 mRNA were related to lower incidence of allergic diseases [42, 43]. TLR2 ligands were also able to inhibit Th2 responses in house dust mite allergic patients [44] and TLR4 signaling by LPS suppressed asthmalike responses in mice [45]. It was also shown that TLR9 intracellular signaling by $\mathrm{CpG}$ induced the release of Th1 cytokines [46] and promoted IgG2a switch, which is related to Th1 pattern [47]. Although our results do not show a clear Th1 pattern in Protocol 2, we hypothesize that the Th2 suppression could also be related to higher expression of TLRs by APCs in P. acnes group.

On the other hand, PS treatment did not induce distinct patterns of TLRs levels between Protocols 1 and 2, as clearly shown in Table 1(b). Therefore, we believe that the effects induced by PS in Th2 potentiation or suppression may be only related to the expression of costimulatory molecules, while the effects induced by $P$. acnes involve a more complex mechanism, including not only the expression of costimulatory molecules, but also the levels of TLRs. However, we cannot exclude other molecules and receptors which were not analyzed in the present work.

The modulation of Th1 and Th2 responses by $P$. acnes and PS was also analyzed by intracellular staining of IL-4 and IL-12 on APCs. In Protocol 1, P. acnes and PS treatments increased the number of IL- $4^{+}$APCs and decreased the number of IL-12 $2^{+}$(Figure 4(a)), confirming the potentiation of Th2 response. In Protocol 2, there was little increase of IL$4^{+}$cells and also increase of IL-12 $2^{+}$, indicating the suppression of Th2 response, but not changing to a Th1 pattern, since the number of IL- $4^{+}$cells remained higher than the number of IL-12 ${ }^{+}$(Figure 4(e)). The production level of these cytokines by APCs did not show a defined pattern when comparing the two protocols (Figure 4). We believe that, in our model, maybe the modulation of the intracellular levels of cytokines in APCs is not as relevant as the number of producing cells. The most abundant APC population in spleen is composed of B lymphocytes, which highly impacted the total number of APCs (data not shown). Interestingly, MFI of IL-12 ${ }^{+}$B lymphocytes is decreased in $P$. acnes treatment according to Protocol 1 (Figure 4(b)) and increased in Protocol 2 (Figure $4(\mathrm{f})$ ). The same is not observed in PS groups, probably because the whole bacterium has other components that can be responsible for this specific effect.

Although the production of IL- 4 and IL-12 by spleen APCs does not show a clear Th1 pattern in Protocol 2, we have already demonstrated the induction of intracellular and serum IFN- $\gamma$ in mice treated with $P$. acnes according to this protocol [24]. Another important effect of P. acnes is its capacity to induce the production of IL-18 [11]. Therefore, maybe the combination of IL-12 and IL-18 production by APCs could be responsible for directing the $\mathrm{T}$ helper response, resulting in the release of IFN $-\gamma$. The production of IL-18 in this model needs further investigation.

Another essential cytokine in this model is undoubtedly IL-5, whose production was analyzed in the total spleen population, certainly including IL-5-producing T lymphocytes, which are the main IL-5 producer cells. The analysis of IL-5 ${ }^{+}$ spleen cells strongly correlated to previous data, since $P$. acnes and PS increased the number of IL- $5^{+}$cells in Protocol 1 (Th2 potentiation) and decreased in Protocol 2 (Th2 suppression) (Figure 5). IL-5 induces maturation, survival, and chemoattraction of eosinophils and enhances their effector functions, playing a pivotal role in allergic inflammation $[36,37]$. In fact, in our previous data, $P$. acnes and PS treatments had a great impact on eosinophilia in the type I hypersensitivity model, inducing enhanced eosinophil infiltration in Protocol 1 and inhibiting it in Protocol $2[24,26]$.

Then, even with little differences in the production of IL4 and IL-12 by APCs, both protocols induced significant differences on IL-5 production and eosinophilia. We believe IL5 is an essential cytokine involved with the biological effects observed in this model, while IL- 4 and IL-12 production by APCs might not be an essential factor for potentiation or suppression of the Th2 response. Therefore, in this model, the differential expression of costimulatory molecules and TLRs by $B$ lymphocytes, macrophages, and dendritic cells seems to be more relevant for Th1/Th2 direction by P. acnes and PS than the production of cytokines by these cells.

Our results suggest that APCs can be directly modulated by $P$. acnes and PS in this model, which is in agreement with other studies showing direct activation of innate immune cells by $P$. acnes via TLR2 and TLR9 $[15,16]$. In order to confirm this hypothesis of direct activation of APCs, we differently stimulated BMDC with $P$. acnes, PS, and/or OVA and cocultured these cells with OVA-primed T lymphocytes, analyzing the pattern of cytokines released in the culture supernatants. Interestingly, only IL-5 and IL-17 were detected in these cultures, although we have shown the production of IL- 4 and IL-12 by APCs in Figure 4. It is clear for us that these cocultures did not represent exactly the same conditions obtained in vivo, concerning the microenvironment and treatment periods, for example, which can explain the absence of IL- 4 and IL-12 in vitro. Besides, it is not reasonable to compare the levels of intracellular cytokines, which may not necessarily be released, with the levels of cytokines in culture supernatants. Therefore, a better analysis of in vitro results should include the intracellular cytokines staining, which needs further investigation.

Both IL-5 and IL-17 were increased in the in vitro potentiation protocol (Protocol 1-like) and decreased in the 
in vitro suppression protocol (Protocol 2-like) (Figure 6). As mentioned above, IL-5 is involved with eosinophilia and plays an essential role in allergic inflammation $[36,37]$. On the other hand, IL-17 has been implicated in noncanonical forms of asthma, where neutrophilia is more evident than eosinophilia [48]. Interestingly, in our previous studies using this hypersensitivity model, although eosinophilia in control (saline) groups is considered intense, these cells are not the only population present in the cellular infiltrate $[24,26]$, and the remaining cells are mainly neutrophils (data not shown). This may be an indication of the participation of IL-17 in this model. In fact, clinical observations have suggested the coexistence of both Th2 and Th17 phenotypes in the same patient [48], and a new population of $\mathrm{CD} 4^{+} \mathrm{T}$ lymphocytes which produces both IL- 4 and IL-17 has been recently identified [49]. Therefore, it is possible that our model could induce both patterns, and future functional studies will certainly contribute to better understand the roles of IL- 5 and IL-17 in this model.

\section{Conclusions}

Our data indicate that $P$. acnes can directly act on antigenpresenting cells, mainly modulating the expression of costimulatory molecules and TLRs, and that PS is an important bacterium compound involved in these immunomodulatory effects.

In our model, this differential expression of costimulatory molecules and TLRs by APCs seems to be essential, rather than the production of IL- 4 and IL-12, for driving the T cell response. The modulation of APCs by $P$. acnes and PS results in different levels of cytokines produced by $\mathrm{T}$ helper cells, probably including IL-5 and IL-17, which seem to be major cytokines in this hypersensitivity model. Ultimately, different patterns of T helper responses are, then, induced, resulting in all the biological effects observed in our previous works using this model.

These results provide new and important contribution to understanding the mechanisms by which $P$. acnes and PS modulate the immune response to OVA in a type I hypersensitivity murine model.

\section{Conflict of Interests}

The authors declare that there is no conflict of interests regarding the publication of this paper.

\section{Acknowledgments}

This work was supported by Grants from FAPESP (2004/ 10354-5; 2014/23593-0), CNPq, and CAPES.

\section{References}

[1] J. J. Leyden, K. J. McGinley, O. H. Mills, and A. M. Kligman, "Age related changes in the resident bacterial flora of the human face," Journal of Investigative Dermatology, vol. 65, no. 4, pp. 379-381, 1975.
[2] J. J. Leyden, K. J. McGinley, and B. Vowels, "Propionibacterium acnes colonization in acne and nonacne," Dermatology, vol. 196, no. 1, pp. 55-58, 1998.

[3] C. Abe, K. Iwai, R. Mikami, and Y. Hosoda, "Frequent isolation of propionibacterium acnes from sarcoidosis lymph nodes," Zentralblatt für Bakteriologie Mikrobiologie und Hygiene A, vol. 256, no. 4, pp. 541-547, 1984.

[4] S. Green, A. Dobrjansky, M. A. Chiasson, E. Carswell, M. K. Schwartz, and L. J. Old, "Corynebacterium parvum as the priming agent in the production of tumor necrosis factor in the mouse," Journal of the National Cancer Institute, vol. 59, no. 5, pp. 1519-1522, 1977.

[5] B. N. Halpern, R. Prévot, G. Biozzi et al., "Stimulation de l'activité phagocytaire du système reticuloendothélial provoquée par Corynebacterium parvum," Journal of the Reticuloendothelial Society, vol. 1, pp. 77-96, 1963.

[6] B. N. Halpern, G. Biozzi, C. Stiffel, and D. Mouton, "Inhibition of tumour growth by administration of killed Corynebacterium parvum," Nature, vol. 212, no. 5064, pp. 853-854, 1966.

[7] R. S. Nussenzweig, "Increased nonspecific resistance to malaria produced by administration of killed Corynebacterium parvum," Experimental Parasitology, vol. 21, no. 2, pp. 224-231, 1967.

[8] Z. Brener and J. E. Cardoso, "Nonspecific resistance against Trypanosoma cruzi enhanced by Corynebacterium parvum," Journal of Parasitology, vol. 62, no. 4, pp. 645-646, 1976.

[9] G. W. Warr and K. James, "Effects of Corynebacterium parvum on the class and subclass of antibody produced in the response of different strains of mice to sheep erythrocytes," Immunology, vol. 28, no. 3, pp. 431-442, 1975.

[10] S. R. Smith, A. Calzetta, J. Bankowski, L. Kenworthy-Bott, and C. Terminelli, "Lipopolysaccharide-induced cytokine production and mortality in mice treated with Corynebacterium parvum," Journal of Leukocyte Biology, vol. 54, no. 1, pp. 23-29, 1993.

[11] H. Okamura, H. Tsutsui, T. Komatsu et al., "Cloning of a new cytokine that induces IFN- $\gamma$ production by T cells," Nature, vol. 378, no. 6552, pp. 88-91, 1995.

[12] T. Dao, K. Ohashi, T. Kayano, M. Kurimoto, and H. Okamura, "Interferon- $\gamma$-inducing factor, a novel cytokine, enhances Fas ligand-mediated cytotoxicity of murine T helper 1 cells," Cellular Immunology, vol. 173, no. 2, pp. 230-235, 1996.

[13] K. Matsui, T. Yoshimoto, H. Tsutsui et al., "Propionibacterium acnes treatment diminishes CD4+NK1.1+ T cells but induces type I T cells in the liver by induction of IL-12 and IL-18 production from Kupffer cells," Journal of Immunology, vol. 159, no. 1, pp. 97-106, 1997.

[14] H. Tsuji, N. Mukaida, A. Harada et al., "Alleviation of lipopolysaccharide-induced acute liver injury in Propionibacterium aches-primed IFN- $\gamma$-deficient mice by a concomitant reduction of TNF- $\alpha$, IL-12, and IL-18 production," The Journal of Immunology, vol. 162, no. 2, pp. 1049-1055, 1999.

[15] J. Kim, M. T. Ochoa, S. R. Krutzik et al., "Activation of toll-like receptor 2 in acne triggers inflammatory cytokine responses," Journal of Immunology, vol. 169, no. 3, pp. 1535-1541, 2002.

[16] C. Kalis, M. Gumenscheimer, N. Freudenberg et al., "Requirement for TLR9 in the immunomodulatory activity of Propionibacterium acnes," The Journal of Immunology, vol. 174, no. 7, pp. 4295-4300, 2005.

[17] A. Velayudham, I. Hritz, A. Dolganiuc, P. Mandrekar, E. KurtJones, and G. Szabo, "Critical role of Toll-like receptors and the common TLR adaptor, MyD88, in induction of granulomas and liver injury," Journal of Hepatology, vol. 45, no. 6, pp. 813-824, 2006. 
[18] S. Jugeau, I. Tenaud, A. C. Knol et al., "Induction of toll-like receptors by Propionibacterium acnes," British Journal of Dermatology, vol. 153, no. 6, pp. 1105-1113, 2005.

[19] L. Romics Jr., A. Dolganiuc, K. Kodys et al., "Selective priming to toll-like receptor 4 (TLR4), not TLR2, ligands by P. acnes involves up-regulation of MD-2 in mice," Hepatology, vol. 40, no. 3, pp. 555-564, 2004.

[20] B. Sahdo, E. Särndahl, F. Elgh, and B. Söderquist, "Propionibacterium acnes activates caspase-1 in human neutrophils," Acta Pathologica, Microbiologica et Immunologica Scandinavica, vol. 121, no. 7, pp. 652-663, 2013.

[21] A. S. MacDonald, A. D. Straw, B. Bauman, and E. J. Pearce, "CD8 ${ }^{-}$dendritic cell activation status plays an integral role in influencing Th2 response development," The Journal of Immunology, vol. 167, no. 4, pp. 1982-1988, 2001.

[22] A. S. Macdonal, A. D. Straw, N. M. Dalto, and E. J. Pearce, "Cutting edge: Th2 response induction by dendritic cells: a role for CD401," Journal of Immunology, vol. 168, no. 2, pp. 537-540, 2002.

[23] L. Cervi, A. S. MacDonald, C. Kane, F. Dzierszinski, and E. J. Pearce, "Cutting edge: dendritic cells copulsed with microbial and helminth antigens undergo modified maturation, segregate the antigens to distinct intracellular compartments, and concurrently induce microbe-specific Th1 and helminth -specific Th2 responses," The Journal of Immunology, vol. 172, no. 4, pp. 20162020, 2004

[24] E. G. Braga, R. Z. Ananias, J. S. Mussalem et al., "Treatment with Propionibacterium acnes modulates the late phase reaction of immediate hypersensitivity in mice," Immunology Letters, vol. 88, no. 2, pp. 163-169, 2003.

[25] S. Facincone, A. L. Pereira De Siqueira, S. Jancar, M. Russo, J. A. Marzagão Barbuto, and M. Mariano, "A novel murine model of late-phase reaction of immediate hypersensitivity," Mediators of Inflammation, vol. 6, no. 2, pp. 127-133, 1997.

[26] C. C. Squaiella, A. L. F. Longhini, E. G. Braga et al., "Modulation of the type I hypersensitivity late phase reaction to OVA by Propionibacterium acnes-soluble polysaccharide," Immunology Letters, vol. 121, no. 2, pp. 157-166, 2008.

[27] J. S. Mussalem, J. R. C. Vasconcelos, C. C. Squaiella et al., "Adjuvant effect of the Propionibacterium acnes and its purified soluble polysaccharide on the immunization with plasmidial DNA containing a Trypanosoma cruzi gene," Microbiology and Immunology, vol. 50, no. 4, pp. 253-263, 2006.

[28] R. Z. Ananias, E. G. Rodrigues, E. G. Braga et al., "Modulatory effect of killed Propionibacterium acnes and its purified soluble polysaccharide on peritoneal exudate cells from C57Bl/6 mice: major NKT cell recruitment and increased cytotoxicity," Scandinavian Journal of Immunology, vol. 65, no. 6, pp. 538-548, 2007.

[29] C. C. Squaiella, R. Z. Ananias, J. S. Mussalem et al., "In vivo and in vitro effect of killed Propionibacterium acnes and its purified soluble polysaccharide on mouse bone marrow stem cells and dendritic cell differentiation," Immunobiology, vol. 211, no. 1-2, pp. 105-116, 2006.

[30] V. O. Reis, J. C. Silva, G. T. Souza et al., "The polysaccharide fraction of Propionibacterium acnes modulates the development of experimental focal segmental glomerulosclerosis," Immunobiology, vol. 217, no. 9, pp. 831-841, 2012.

[31] M. M. Bradford, "A rapid and sensitive method for the quantitation of microgram quantities of protein utilizing the principle of protein dye binding," Analytical Biochemistry, vol. 72, no. 1-2, pp. 248-254, 1976.
[32] J. W. Palmer and T. D. Gerlough, "A simple method for preparing antigenic substances from the typhoid bacillus," Science, vol. 92, no. 2381, pp. 155-156, 1940.

[33] M. Dubois, K. Gilles, J. K. Hamilton, P. A. Rebers, and F. Smith, "A colorimetric method for the determination of sugars," Nature, vol. 168, no. 4265, p. 167, 1951.

[34] P. Dasari, I. C. Nicholson, G. Hodge, G. W. Dandie, and H. Zola, "Expression of toll-like receptors on B lymphocytes," Cellular Immunology, vol. 236, no. 1-2, pp. 140-145, 2005.

[35] J. S. Mussalem, C. C. Squaiella-Baptistão, D. Teixeira et al., "Adjuvant effect of killed propionibacterium acnes on mouse peritoneal B-1 lymphocytes and their early phagocyte differentiation," PLoS ONE, vol. 7, no. 3, Article ID e33955, 2012.

[36] A. F. Lopez, C. J. Sanderson, J. R. Gamble, H. D. Campbell, I. G. Young, and M. A. Vadas, "Recombinant human interleukin 5 is a selective activator of human eosinophil function," The Journal of Experimental Medicine, vol. 167, no. 1, pp. 219-224, 1988.

[37] Y. Yamaguchi, Y. Hayashi, Y. Sugama et al., "Highly purified murine interleukin 5 (IL-5) stimulates eosinophil function and prolongs in vitro survival. IL-5 as an eosinophil chemotactic factor," Journal of Experimental Medicine, vol. 167, no. 5, pp. $1737-1742,1988$

[38] D. M. Lindell, A. A. Berlin, M. A. Schaller, and N. W. Lukacs, "B cell antigen presentation promotes Th2 responses and immunopathology during chronic allergic lung disease," PLoS ONE, vol. 3, no. 9, Article ID e3129, 2008.

[39] C. P. M. Broeren, G. S. Gray, B. M. Carreno, and C. H. June, "Costimulation light: activation of $\mathrm{CD} 4^{+} \mathrm{T}$ cells with $\mathrm{CD} 80$ or CD86 rather than anti-CD28 leads to a Th2 cytokine profile," Journal of Immunology, vol. 165, no. 12, pp. 6908-6914, 2000.

[40] L. S. Van Rijt, N. Vos, M. Willart et al., "Essential role of dendritic cell CD80/CD86 costimulation in the induction, but not reactivation, of $\mathrm{TH} 2$ effector responses in a mouse model of asthma," Journal of Allergy and Clinical Immunology, vol. 114, no. 1, pp. 166-173, 2004.

[41] D. J. Lenschow, T. L. Walunas, and J. A. Bluestone, "CD28/B7 system of T cell costimulation," Annual Review of Immunology, vol. 14, pp. 233-258, 1996.

[42] C. Braun-Fahrländer, J. Riedler, U. Herz et al., "Environmental exposure to endotoxin and its relation to asthma in school-age children," The New England Journal of Medicine, vol. 347, no. 12, pp. 869-877, 2002.

[43] R. P. Lauener, T. Birchler, J. Adamski et al., "Expression of CD14 and Toll-like receptor 2 in farmers' and non-farmers' children," The Lancet, vol. 360, no. 9331, pp. 465-466, 2002.

[44] R. C. Taylor, P. Richmond, and J. W. Upham, “Toll-like receptor 2 ligands inhibit TH2 responses to mite allergen," The Journal of Allergy and Clinical Immunology, vol. 117, no. 5, pp. 1148-1154, 2006.

[45] D. Rodríguez, A. C. Keller, E. L. Faquim-Mauro et al., "Bacterial lipopolysaccharide signaling through Toll-like receptor 4 suppresses asthma-like responses via nitric oxide synthase 2 activity," The Journal of Immunology, vol. 171, no. 2, pp. 1001-1008, 2003.

[46] S. Bauer, D. Hangel, and P. Yu, "Immunobiology of toll-like receptors in allergic disease," Immunobiology, vol. 212, no. 6, pp. 521-533, 2007.

[47] A. Jegerlehner, P. Maurer, J. Bessa, H. J. Hinton, M. Kopf, and M. F. Bachmann, "TLR9 signaling in B cells determines class switch recombination to IgG2a," The Journal of Immunology, vol. 178, no. 4, pp. 2415-2420, 2007. 
[48] L. Cosmi, F. Liotta, E. Maggi, S. Romagnani, and F. Annunziato, "Th17 cells: new players in asthma pathogenesis," Allergy, vol. 66, no. 8, pp. 989-998, 2011.

[49] L. Cosmi, L. Maggi, V. Santarlasci et al., "Identification of a novel subset of human circulating memory $\mathrm{CD}^{+} \mathrm{T}$ cells that produce both IL-17A and IL-4," Journal of Allergy and Clinical Immunology, vol. 125, no. 1-3, pp. 222.e4-230.e4, 2010. 


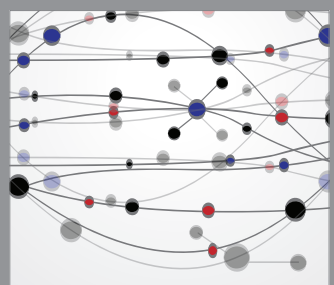

The Scientific World Journal
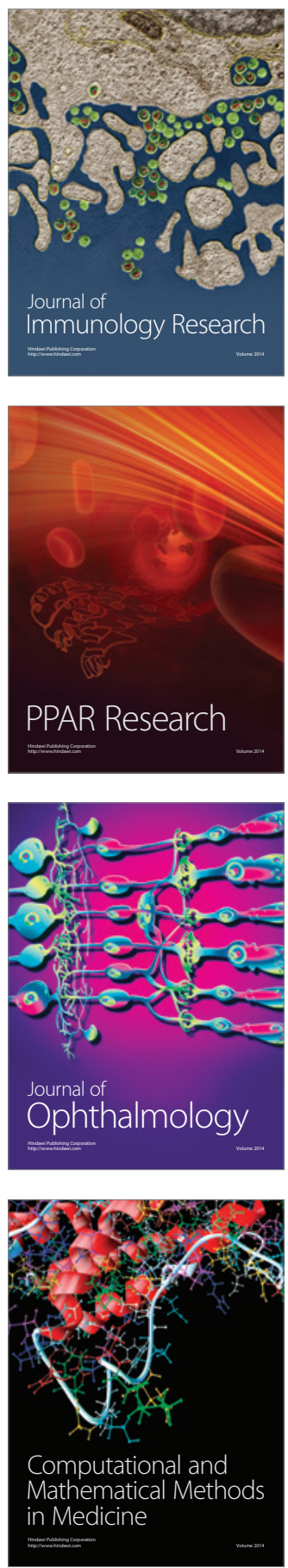

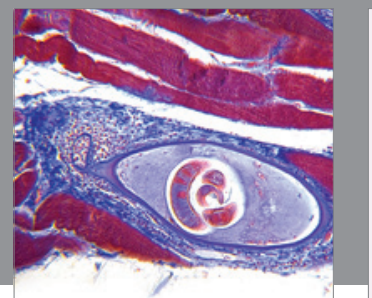

Gastroenterology

Research and Practice
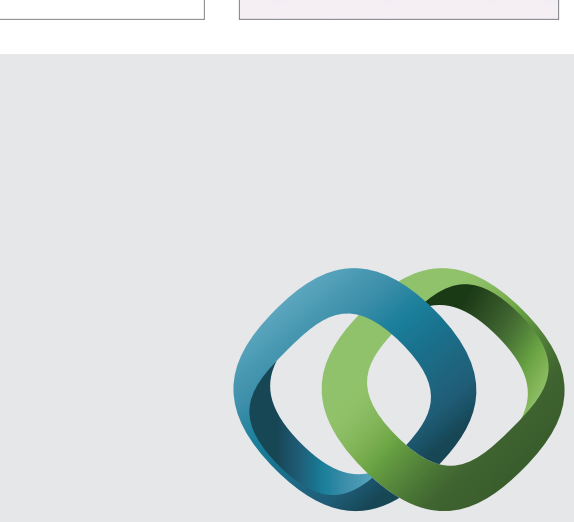

\section{Hindawi}

Submit your manuscripts at

http://www.hindawi.com
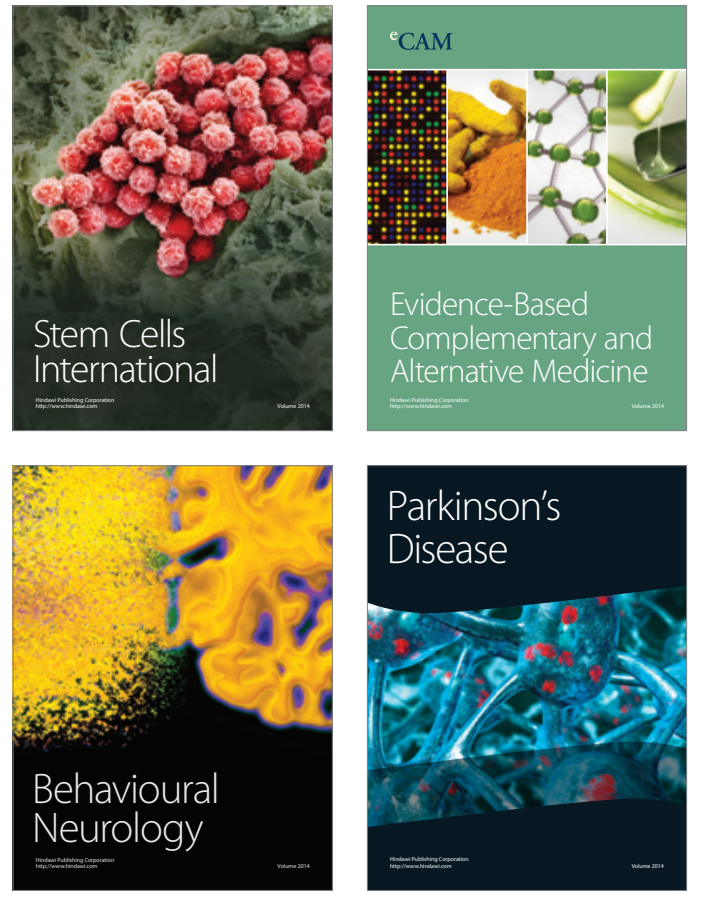
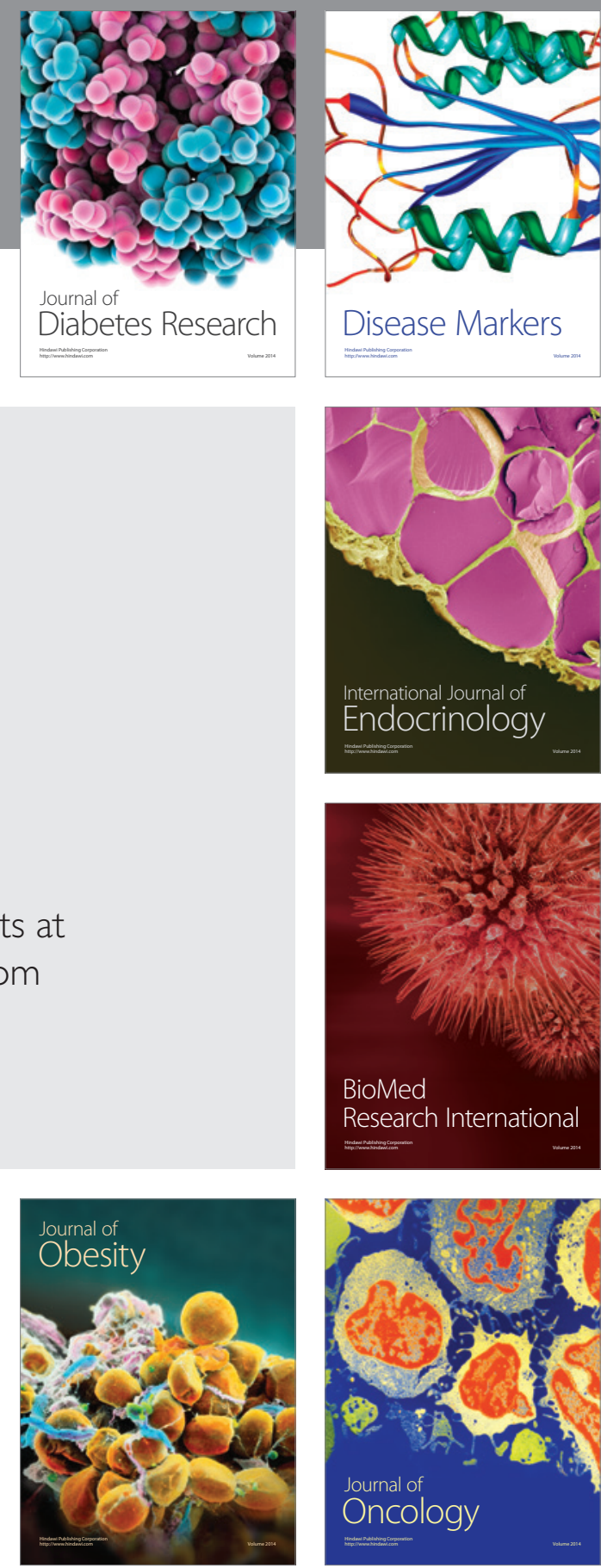

Disease Markers
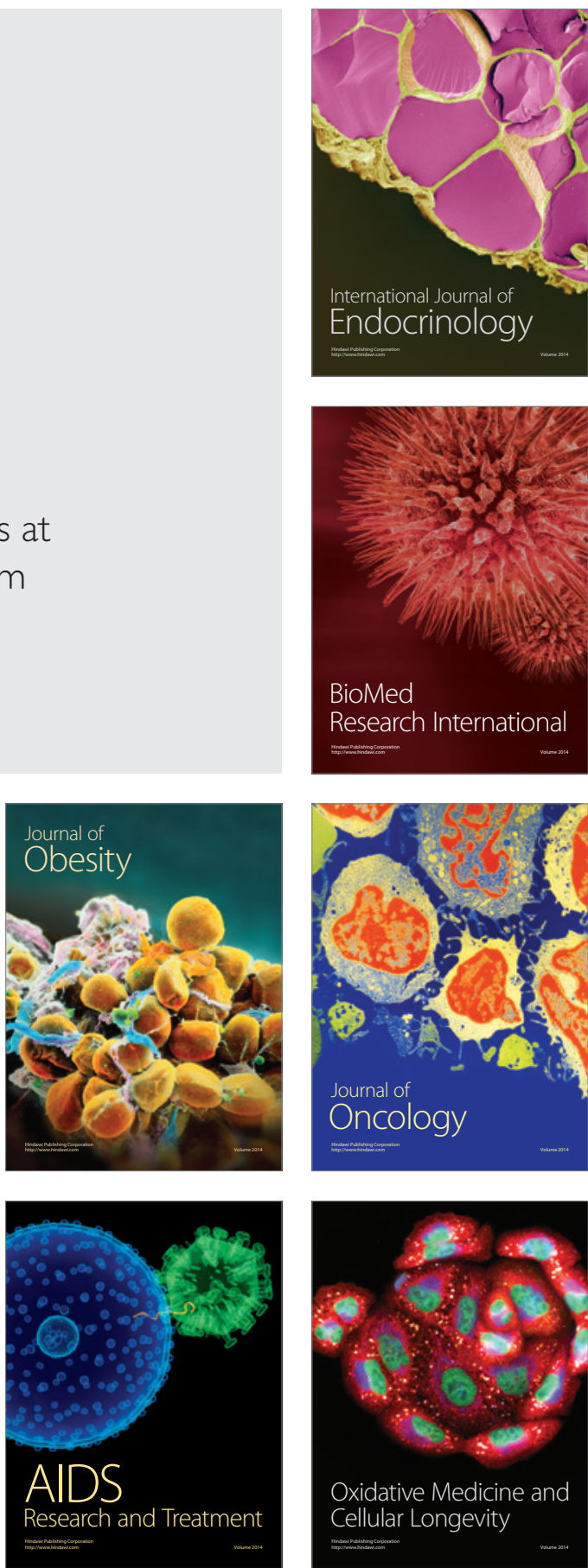